\title{
EFEITO DE MICROONDAS NA ESTRUTURA CRISTALINA E NA ATIVIDADE CATALÍTICA DE ARGILAS
}

\author{
Angelo C. Pinto \\ Departamento de Química Orgânica, Instituto de Química, Universidade Federal do Rio de Janeiro, CT, Bloco A, Ilha do \\ Fundão, 21945-970 Rio de Janeiro - RJ, Brasil \\ Cristiano Honório de Oliveira \\ Petróleo Brasileiro S/A, Recursos Humanos, Universidade Petrobras, Rua General Canabarro, 500, 20271-900 \\ Rio de Janeiro-RJ, Brasil \\ Núbia Moura Ribeiro* \\ Centro Federal de Educação Tecnológica da Bahia, Rua Emídio dos Santos, s/n, 40301-015 Salvador - BA, Brasil \\ Recebido em 14/3/07; aceito em 6/9/07; publicado na web em 26/2/08
}

\begin{abstract}
THE EFFECT OF MICROWAVE IRRADIATION ON THE CRYSTALLINE STRUCTURE AND CATALYTIC ACTIVITY OF CLAYS. The effect of microwave (MW) irradiation on the crystalline structure of two natural clays and one commercial clay, Montmorillonite $\mathrm{K} 10$, was analyzed comparing the X-ray diffraction, $\mathrm{N}_{2}$ isotherms, NMR-MAS of ${ }^{27} \mathrm{Al}$ and ${ }^{29} \mathrm{Si}$ spectra of the clays before and after MW irradiation. The preparation of dioxolane ketals of isatin was used to analyze the MW effect on the catalyst activation. The yields achieved using catalysts activated by MW irradiation were lower (2 to 5\%) than the yields achieved using catalysts activated by heat in a conventional oven.
\end{abstract}

Keywords: clays; microwave; catalysis.

\section{INTRODUÇÃO}

Microondas (MO) estão se tornando uma fonte de energia cada vez mais presente em laboratórios de química e na ativação de reações orgânicas. ${ }^{1}$ As reações podem ser realizadas em forno de microondas doméstico ou em reatores especificamente desenhados para esta finalidade. ${ }^{2,3} \mathrm{~A}$ absorção seletiva de energia na faixa de microondas por moléculas polares produz uma aceleração significante nas reações, reduzindo o tempo reacional; ressalta-se que moléculas apolares não respondem aos mecanismos de aquecimento por microondas. As microondas são radiações eletromagnéticas não ionizantes na faixa de freqüência de 300 a 300.000 $\mathrm{MHz}$, que correspondem a comprimentos de onda de $1 \mathrm{~mm}$ a $1 \mathrm{~m}$. A região de microondas situa-se entre a região de infravermelho e a das ondas de rádio no espectro eletromagnético. ${ }^{4}$

Paralelamente à aplicação de microondas como fonte energética, a última década testemunhou a crescente utilização de catálise heterogênea em reações orgânicas, ${ }^{5}$ tendo as argilas sido empregadas com sucesso. ${ }^{6,7}$ A grande área específica das argilas em conjunto com propriedades específicas, tais como capacidade de troca iônica e de aumento do espaçamento interlamelar, têm sido exploradas para sua aplicação como catalisadores. Com a combinação de microondas e catalisadores sólidos, ampliou-se a possibilidade de realização de reações em condições sem solventes. ${ }^{8,9}$

O uso de argilas como catalisadores requer sua ativação prévia. A ativação é um processo de remoção da água adsorvida na argila, deixando acessíveis sítios catalíticos nos quais moléculas de água poderiam estar ligadas. ${ }^{10} \mathrm{~A}$ ativação geralmente é feita por aquecimento a $120{ }^{\circ} \mathrm{C}$ por $1 \mathrm{~h}$, seguido de resfriamento em dessecador a vácuo. Essa ativação pode ser realizada por outros métodos, como, por exemplo, por aquecimento com microondas. Entretanto, na literatura há poucos estudos sobre o efeito da ativação por microondas nas propriedades catalíticas das argilas e na estrutura de seus componentes. ${ }^{11}$

\footnotetext{
*e-mail: nubia@cefetba.br
}

Neste trabalho foi analisado o efeito de microondas na estrutura cristalina de três argilas e na sua atividade catalítica. Foram analisadas uma argila comercial, a Montmorilonita K10 (Aldrich), e duas argilas naturais: uma proveniente da Bahia - $\mathbf{A B}$ - e outra da Paraíba - AP. As argilas K10 e AP foram utilizadas sem serem fracionadas ou tratadas com ácidos. A argila $\mathbf{A B}$, recebida em blocos compactados, foi fracionada e tratada com ácido para aumentar sua atividade catalítica.

Especialmente as montmorilonitas são utilizadas como catalisadores por sua alta capacidade de troca catiônica, por serem expansíveis e por apresentarem sítios ácidos de Bronsted e Lewis. Embora a Montmorilonita K10 (Aldrich) seja um material que não apresente fases cristalinas passíveis de comparação com as demais argilas - pois as fases cristalinas são destruídas durante a produção industrial do catalisador - este material foi testado por sua larga aplicação em reações catalíticas com uso de microondas. ${ }^{4,6,8,12} \mathrm{~A}$ acidez de Lewis da $\mathbf{K 1 0}$ é tão eficiente quanto a do $\mathrm{AlCl}_{3}$ e é capaz de promover reações de Friedel-Crafts. ${ }^{13}$

\section{RESULTADOS E DISCUSSÃO}

Como parâmetro de avaliação da quantidade de energia utilizada na irradiação de microondas, foi determinada a potência do forno doméstico utilizado.

Para avaliar o efeito da irradiação de microondas na estrutura cristalina das argilas, fez-se avaliação textural, análise dos difratogramas de raios $\mathrm{X}$ e dos espectros de ressonância magnética nuclear no estado sólido de ${ }^{27} \mathrm{Al}$ e ${ }^{29} \mathrm{Si}$ das argilas com e sem irradiação de microondas. Como dado adicional para caracterização dos catalisadores, obteve-se os valores de capacidade de troca catiônica das argilas não irradiadas com microondas e a análise dos elementos constituintes dessas argilas por fluorescência de raios X.

Para avaliar o efeito da ativação catalítica das argilas por microondas utilizou-se a reação de cetalização da isatina com etilenoglicol. A reação foi realizada com os catalisadores sem ativação, com os catalisadores ativados termicamente e ativados por microondas. 


\section{Determinação da potência de forno de microondas}

Para ativação das argilas e para realização das reações de cetalização, foi utilizado o aparelho de microondas doméstico marca Brastemp, modelo JetDefrost com capacidade de $27 \mathrm{~L}$, que permite irradiação de potência em 10 níveis (1 a 10).

A determinação da potência do forno de microondas foi realizada pela medida indireta da elevação da temperatura da água, pelo aquecimento por um tempo estabelecido. ${ }^{14,15}$ Os resultados obtidos estão apresentados na Tabela 1 . $\mathrm{O}$ valor correspondente à potência máxima $(569,9 \mathrm{~W})$, apesar de estar dentro da faixa de potência típica de fornos de MO domésticos, é consideravelmente inferior à indicada pelo fabricante $(900 \mathrm{~W})$.

Tabela 1. Determinação da potência de forno microondas, usando $1 \mathrm{~L}$ de água por $120 \mathrm{~s}$

\begin{tabular}{lcc}
\hline $\begin{array}{l}\text { Nível de } \\
\text { potência do } \\
\text { aparelho }\end{array}$ & $\begin{array}{c}\text { Variação de } \\
\text { temperatura } \\
\left({ }^{\circ} \mathrm{C}\right)\end{array}$ & $\begin{array}{c}\text { Potência } \\
\text { calculada } \\
(\mathrm{W})\end{array}$ \\
\hline 1 & 4,2 & 72,7 \\
2 & 7,7 & 133,7 \\
3 & 11,8 & 206,4 \\
4 & 14,2 & 247,1 \\
5 & 18,5 & 322,1 \\
6 & 21,0 & 366,7 \\
7 & 23,7 & 412,9 \\
8 & 27,5 & 497,7 \\
9 & 31,3 & 546,6 \\
10 & 32,7 & 569,9 \\
\hline
\end{tabular}

Irradiação das argilas com microondas

As argilas AB, AP e K10 foram irradiadas com microondas por $10 \mathrm{~min}$, sem solvente, na potência máxima do aparelho (569,9 W). Em seguida foram colocadas em dessecador a vácuo para posterior análise ou utilização nas reações de cetalização.

\section{Análise textural das argilas}

A acessibilidade dos reagentes aos sítios ativos é fundamental para a seleção de materiais sólidos utilizados como catalisadores heterogêneos. Por isso, a avaliação textural é de grande importância em catalisadores sólidos. Essa avaliação é feita através das informações obtidas a partir das isotermas de adsorção-dessorção na temperatura de $\mathrm{N}_{2}$ líquido. As isotermas de adsorção/dessorção de $\mathrm{N}_{2}$ da argila da Bahia fracionada e tratada com ácido $(\mathbf{A B})$ e da argila da Bahia fracionada, tratada com ácido e irradiada com microondas (ABMO) apresentaram semelhança entre si (Figura 1). São isotermas do tipo II, que correspondem a materiais com poros principalmente na faixa de mesoporos (20 a $50 \AA$ ) e macroporos (50 $\mathrm{\AA}$ ou maiores). ${ }^{16} \mathrm{O}$ formato do "loop" de histerese indicou poros do tipo fenda. ${ }^{17}$

A faixa de linearidade das isotermas de adsorção/dessorção de $\mathrm{N}_{2}$ das argilas AB e ABMO se enquadrou na faixa de pressões relativas para a qual o método BET é indicado $(0,03$ a 0,35$) .^{7}$ Os dados de análise textural dessas argilas estão mostrados na Tabela 2.

A constante $C$ para cálculos de área específica das argilas $\mathbf{A B}$ e ABMO apresentou valores de 195,2 e 167,7, respectivamente. Esses valores encontram-se na faixa esperada para materiais em que o método BET se aplica (C entre 50 a 300). ${ }^{18}$

As isotermas de adsorção/dessorção de $\mathrm{N}_{2}$ da argila da Paraíba in natura (AP) e irradiada com microondas (APMO) apresenta- ram o mesmo formato (Figura 2). Também são isotermas do tipo II, que, no caso dessas argilas correspondem a materiais com poros
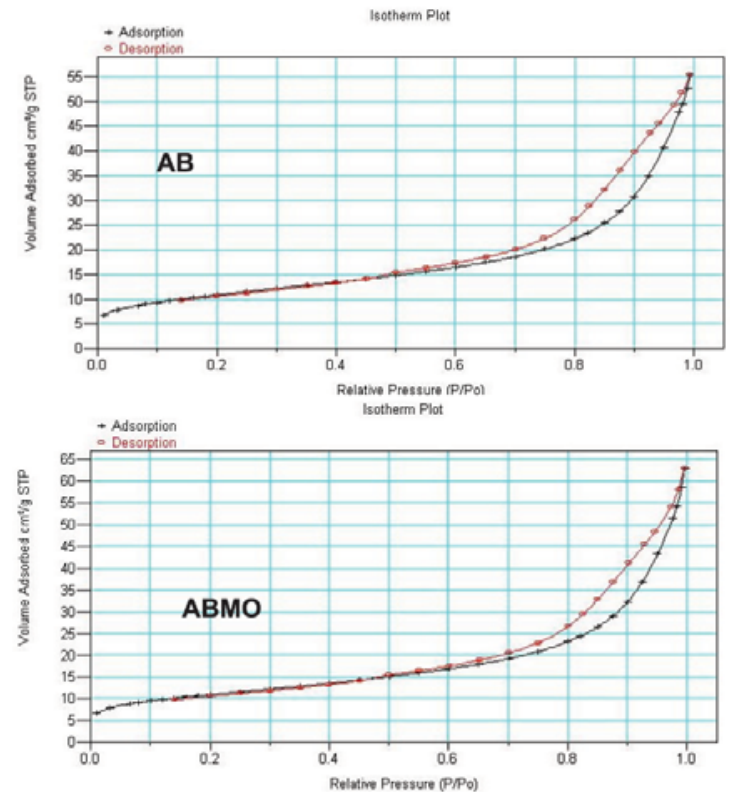

Figura 1. Isotermas de adsorção/dessorção de $N_{2}$ das argilas $A B$ e ABMO

Tabela 2. Dados de análise textural das argilas AB e ABMO

\begin{tabular}{|c|c|c|}
\hline & $\mathbf{A B}$ & ABMO \\
\hline Área específica BET & $41,7 \pm 0,2 \mathrm{~m}^{2} / \mathrm{g}$ & $39,2 \pm 0,1 \mathrm{~m}^{2} / \mathrm{g}$ \\
\hline Constante C & 195,2 & 167,7 \\
\hline Coef. correlação & 0,9999617 & 0,9999785 \\
\hline $\begin{array}{l}\text { Área de poros com diâmetro } \\
\text { entre } 20 \text { e } 1600 \AA \text { (BJH) }\end{array}$ & $38,6 \mathrm{~m}^{2} / \mathrm{g}$ & $37,8 \mathrm{~m}^{2} / \mathrm{g}$ \\
\hline $\begin{array}{l}\text { Volume de poros com diâmetro } \\
\text { entre } 20 \text { e } 1600 \AA \text { ( } \mathrm{BJH})\end{array}$ & $0,097 \mathrm{~cm}^{3} / \mathrm{g}$ & $0,080 \mathrm{~cm}^{3} / \mathrm{g}$ \\
\hline $\begin{array}{l}\text { Diâmetro médio de poros } \\
(4 \mathrm{~V} / \mathrm{A} \text { por BET/BJH) }\end{array}$ & $76,2 / 101,0 \AA$ & $81,1 / 104,8 \AA$ \\
\hline
\end{tabular}
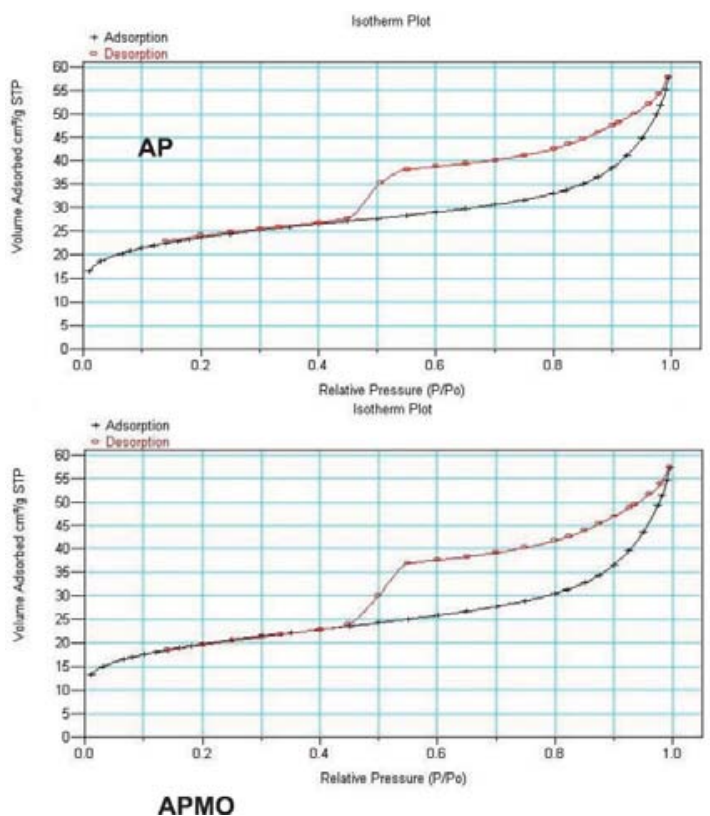

Figura 2. Isotermas de adsorção/dessorção de $N_{2}$ das argilas AP e APMO 
principalmente na faixa de mesoporos (20 a $50 \AA$ ). O formato do loop de histerese indicou poros do tipo fenda. ${ }^{8} \mathrm{~A}$ área específica dessas argilas foi estimada através do método BET e os dados encontrados estão mostrados na Tabela 3.

Tabela 3. Dados da análise textural da argila AP e APMO

\begin{tabular}{|c|c|c|}
\hline & AP & APMO \\
\hline Área específica BET & $82,7 \pm 0,7 \mathrm{~m}^{2} / \mathrm{g}$ & $69,9 \pm 0,4 \mathrm{~m}^{2} / \mathrm{g}$ \\
\hline Constante C & $-720,0$ & 543,0 \\
\hline Coef. correlação & 0,9997844 & 0,9998859 \\
\hline $\begin{array}{l}\text { Área de poros com diâmetro } \\
\text { entre } 20 \text { e } 1600 \AA \text { (BJH) }\end{array}$ & $51,5 \mathrm{~m}^{2} / \mathrm{g}$ & $59,3 \mathrm{~m}^{2} / \mathrm{g}$ \\
\hline $\begin{array}{l}\text { Volume de poros com diâmetro } \\
\text { entre } 20 \text { e } 1600 \AA \text { (BJH) }\end{array}$ & $0,076 \mathrm{~cm}^{3} / \mathrm{g}$ & $0,085 \mathrm{~cm}^{3} / \mathrm{g}$ \\
\hline $\begin{array}{l}\text { Diâmetro médio de poros } \\
\text { (4 V/A por BET/BJH) }\end{array}$ & $37,3 / 58,6 \AA$ & $43,5 / 57,6 \AA$ \\
\hline
\end{tabular}

A constante $\mathrm{C}$ para cálculo de área específica das argilas AP e APMO apresentou valores de $-720,0$ e 543,0, respectivamente. Em geral, para materiais em que o método BET é aplicável, os valores de $\mathrm{C}$ encontram-se na faixa de 50 a 300 . Os valores estão fora da faixa esperada porque as isotermas de $\mathrm{N}_{2}$ dessas argilas não têm linearidade na faixa de pressões relativas de 0,05 e 0,35, e assim o método BET não se aplica satisfatoriamente.

Em resumo, após a irradiação por microondas, as áreas superficiais das argilas, estimadas pelo método BET, sofreram as modificações apresentadas na Tabela 4. Como o erro do método BET é aproximadamente $10 \%$ e as diferenças de áreas encontram-se nessa faixa, não se pode afirmar que houve realmente modificações na textura das argilas pela aplicação de microondas.

Tabela 4. Modificações na área específica das AB e ABMO, AP e APMO

\begin{tabular}{lcccc}
\hline & AB & ABMO & AP & APMO \\
\hline Área específica BET $\left(\mathrm{m}^{2} / \mathrm{g}\right)$ & 41,7 & 39,2 & 82,7 & 69,9 \\
\hline
\end{tabular}

\section{Difratometria de raios $\mathbf{X}$ das argilas}

$\mathrm{O}$ método de difratometria de raios $\mathrm{X}$ oferece a vantagem de apresentar um grande número de picos no difratograma de argilominerais, o que facilita sua identificação, principalmente no caso de misturas, onde pode haver superposição de alguns picos, mas nunca de todos. ${ }^{19}$ Os difratogramas das argilas da Bahia fracionada $(\mathbf{A B F})$, fracionada e tratada com ácido $(\mathbf{A B})$ e fracionada, tratada com ácido e irradiada com microondas (ABMO) são praticamente idênticos, indicando que a irradiação de microondas não modificou o espaçamento basal dos cristais e, portanto, não causou modificação na estrutura cristalina das amostras (Figura 3). Os difratogramas mostram que a argila da Bahia contém ilita como argilomineral mais abundante, além de conter albita, caulinita e quartzo. Ilita é um argilomineral não expansível, que apresenta arranjo dioctaédrico e fórmula geral $\mathrm{K}_{\mathrm{x}}\left(\mathrm{Al}_{2}\right)\left(\mathrm{Si}_{4-\mathrm{x}} \mathrm{Al}_{\mathrm{x}}\right) \mathrm{O}_{10}(\mathrm{OH})_{2}$. Seu espaço interlamelar é de $10 \AA^{20}$

Os difratogramas da argila da Paraíba sem irradiação de microondas (AP) e irradiada com microondas (APMO) também são praticamente idênticos, indicando também que a irradiação de microondas não interferiu na estrutura cristalina das amostras (Figura 4). Os difratogramas mostram que a argila da Paraíba contém montmorilonita como argilomineral mais abundante, além de conter albita e quartzo. Montmorilonita é um argilomineral expansível, que apresenta arranjo dioctaédrico e fórmula geral $\mathrm{M}_{\mathrm{x}}\left(\mathrm{Al}_{2-\mathrm{x}} \mathrm{Mg}_{\mathrm{x}}\right)$

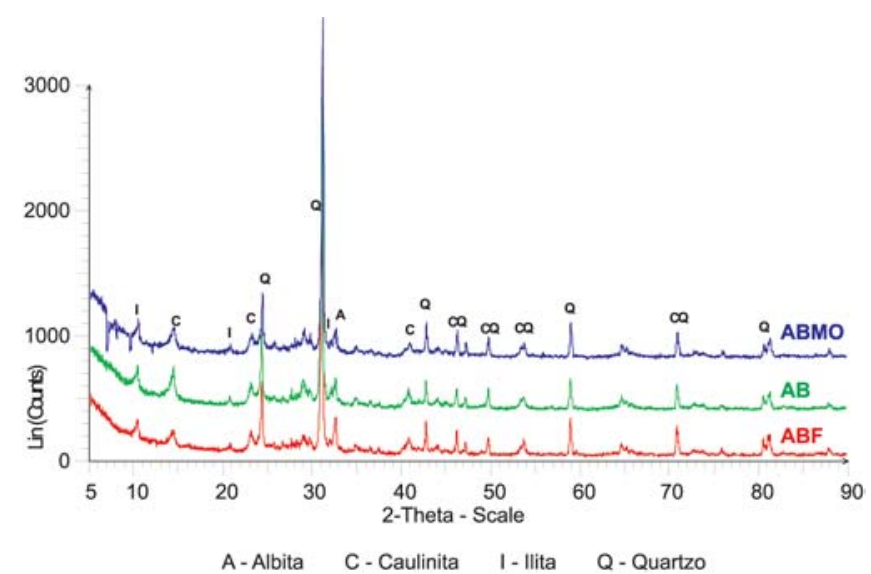

Figura 3. Difratogramas das argilas $A B F, A B$ e $A B M O$

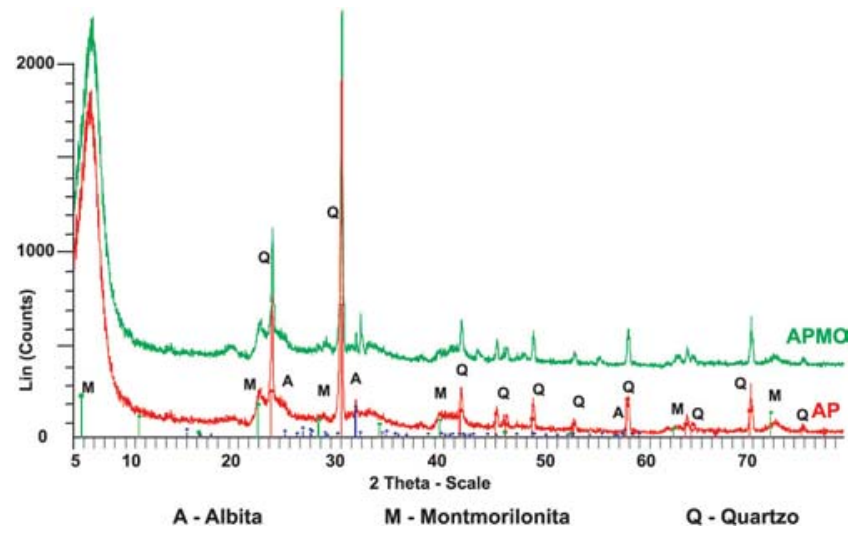

Figura 4. Difratogramas das argilas AP e APMO

$\left(\mathrm{Si}_{4}\right) \mathrm{O}_{10}(\mathrm{OH})_{2} \cdot \mathrm{nH}_{2} \mathrm{O}$, sendo $\mathrm{M}$ os cátions metálicos interlamelares. Seu espaço interlamelar é de $15 \AA \AA^{20}$

Também foram feitos testes quanto ao efeito de microondas sobre a estrutura da argila K10. Para isso, foram obtidos difratogramas da argila K10 fornecida pela Aldrich Co. (K10) e difratogramas da argila tratada com diferentes solventes - hexano (K10-H), acetato de etila (K10-A) ou metanol (K10-M) - e irradiada com microondas em potência mínima $(72,7 \mathrm{~W})$ por $10 \mathrm{~min}$, bem como difratogramas da argila tratada com diferentes solventes - hexano (K10-HPM), acetato de etila (K10-APM) ou metanol (K10-MPM) - e irradiada com microondas em potência máxima $(569,9 \mathrm{~W})$, e da argila sem solvente irradiada em potência máxima (K10-PM) por 10 min. Os difratogramas obtidos mostram-se idênticos (Figura 5). Cabe ressaltar que essa argila praticamente não apresenta sinais correspondentes à fase cristalina montmorilonita.

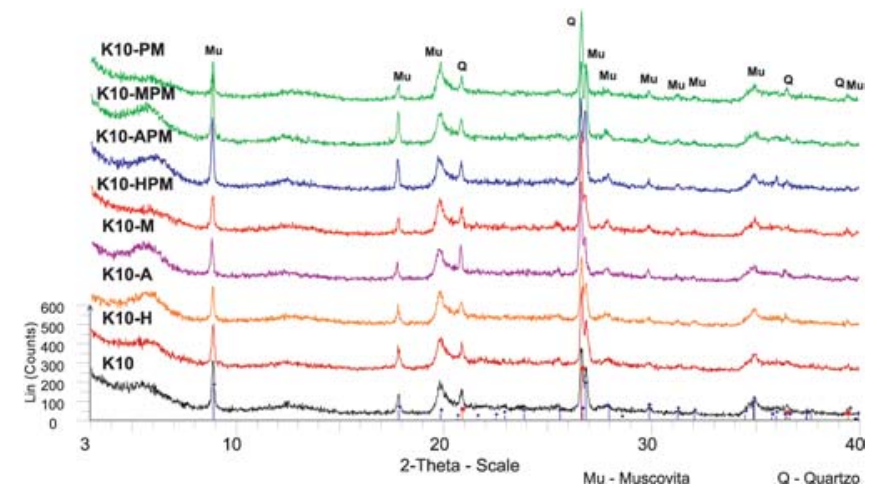

Figura 5. Difratogramas da argila K10, em diferentes condições 
Essa fase cristalina deve ter sido destruída durante o tratamento industrial para produção deste catalisador. ${ }^{12}$

Em síntese, nenhuma das argilas irradiadas com microondas apresentou alterações significativas no padrão de difração das fases cristalinas componentes.

\section{Ressonância magnética nuclear no estado sólido das argilas}

Os espectros de RMN-MAS de ${ }^{27} \mathrm{Al}$ das argilas da Bahia (AB e ABMO) apresentaram baixa resolução devido principalmente às impurezas de feldspato (albita). No caso do espectro da argila ABMO a resolução não foi suficiente para ser analisado.

$\mathrm{O}$ espectro de RMN-MAS de ${ }^{27} \mathrm{Al}$ da APMO apresentou basicamente os mesmos sinais encontrados no espectro de AP; entretanto, o sinal devido ao alumínio tetraédrico, coordenado com 4 átomos de oxigênio ( $\mathrm{Al}-4 \mathrm{O})$, em torno de $50 \mathrm{ppm}$, apresentou menor resolução que no espectro da argila $\mathbf{A P}$ (Tabela 5).

Tabela 5. Deslocamento químico no espectro de RMN-MAS ${ }^{27} \mathrm{Al}$ de $\mathbf{A B}$ e $\mathbf{A P}$

\begin{tabular}{lcc}
\hline Tipo de Alumínio & $\mathbf{A B}$ & $\mathbf{A P}$ \\
\hline $\mathrm{Al}$ octaédrico $(\mathrm{Al}-6 \mathrm{O})$ & $-0,16 \mathrm{ppm}$ & $3,01 \mathrm{ppm}$ \\
$\mathrm{Al}$ tetraédrico $(\mathrm{Al}-4 \mathrm{O})$ & $53,4 \mathrm{ppm}$ & $49,3 \mathrm{ppm}$ \\
\hline
\end{tabular}

No espectro de RMN-MAS de ${ }^{29} \mathrm{Si}$ de argilas são observados deslocamentos químicos negativos (baixa frequiência) em relação ao tetrametilsilano (TMS - referência primária, $0 \mathrm{ppm}$ ), com o aumento da polimerização das unidades Qn, ou seja, a blindagem do átomo central de Si aumenta na seqüência Q0 $<$ Q1 $<$ Q2 $<$ Q3 $<$ Q4, onde os dígitos representam o número de elementos ligados covalentemente aos oxigênios da primeira esfera de coordenação dos silícios ${ }^{21}$ (Figura 6).

\begin{tabular}{|c|c|c|c|c|}
\hline $\begin{array}{c}\text { O- } \\
\text {-O Si O- } \\
\text { O- }\end{array}$ & $\begin{array}{c}\text { O - } \\
\text {-O Si O Si } \\
\text { O - }\end{array}$ & $\begin{array}{c}\text { O- } \\
\text { Si O Si O Si } \\
\text { O- }\end{array}$ & $\begin{array}{c}\mathrm{Si} \\
\mathrm{O} \\
\mathrm{Si} \\
\mathrm{O} \\
\mathrm{Si} \\
\mathrm{O}-\end{array}$ & $\begin{array}{c}S i \\
O \\
\text { Si O Si O Si } \\
\text { O } \\
\text { Si }\end{array}$ \\
\hline $\begin{array}{c}\text { Q0 } \\
\text { (monômero) }\end{array}$ & $\begin{array}{c}\text { Q1 } \\
\text { (final de rede } \\
\text { cristailina) }\end{array}$ & $\begin{array}{l}\mathbf{Q 2} \\
\text { (grupo inter- } \\
\text { mediário na } \\
\text { rede) }\end{array}$ & $\begin{array}{c}\text { Q3 } \\
\text { (grupo } \\
\text { ramificado) }\end{array}$ & $\begin{array}{c}\text { Q4 } \\
\text { (grupo de } \\
\text { junção } \\
\text { cruzada) }\end{array}$ \\
\hline
\end{tabular}

O espectro de RMN-MAS de ${ }^{29} \mathrm{Si}$ da ABMO apresentou-se praticamente idêntico ao espectro da $\mathbf{A B}$, com sinais característicos de caulinita e de quartzo ${ }^{22,23}$ (Tabela 6). O espectro de RMN-MAS de ${ }^{29} \mathrm{Si}$ da APMO apresentou-se praticamente idêntico ao espectro da AP, com sinais característicos de montmorilonita e de quartzo.

Tabela 6. Deslocamento químico no espectro de RMN-MAS ${ }^{29} \mathrm{Si}$ de $\mathbf{A B}$ e $\mathbf{A P}$

\begin{tabular}{lcc}
\hline Tipo de Silício & $\mathbf{A B}$ & $\mathbf{A P}$ \\
\hline Q3 - argila & $-91,3 \mathrm{ppm}$ & $-93,5 \mathrm{ppm}$ \\
Q4 - quartzo & $-107,1 \mathrm{ppm}$ & $-107,0 \mathrm{ppm}$ \\
\hline
\end{tabular}

Outros dados de caracterização das argilas

Capacidade de troca catiônica

A título de reunir mais informações sobre as argilas naturais disponíveis $\mathbf{A B}$ e $\mathbf{A P}$, foram determinadas suas capacidades de troca catiônica (CTC). A quantidade de cátions trocáveis por unidade de peso da argila é denominada CTC. Em geral a CTC é medida em miliequivalentes por $100 \mathrm{~g}$ de argila seca (meq/100 g) e varia com o tipo de argilomineral. As esmectitas têm a maior concentração de cátions entre camadas e, assim, têm a maior capacidade de cátions trocáveis: cerca de 70 a $120 \mathrm{meq} / 100$ g. ${ }^{11}$

Seguiu-se o procedimento proposto por Jackson ${ }^{24}$ e utilizado também por Frenkel e Shainberg ${ }^{25}$ para a determinação da capacidade de troca de cátions de uma montmorilonita. Na Tabela 7 estão apresentados os valores de CTC para a argila da Bahia e para a argila da Paraíba. No caso da argila da Bahia (AB), o valor de $59 \pm$ $2 \mathrm{meg} / 100 \mathrm{~g}$ está um pouco acima da faixa de 10-50 prevista para ilita (micas). Tal desvio deve-se à presença das demais fases. No caso da argila da Paraíba (AP), que contém predominantemente montmorilonita, o valor de $187 \pm 5 \mathrm{meg} / 100 \mathrm{~g}$ está dentro da faixa de $80-200 \mathrm{meq} / 100 \mathrm{~g}$ prevista para este argilomineral.

Tabela 7. Valores de CTC para as argilas da Bahia e da Paraíba

\begin{tabular}{lcc}
\hline Argila & CTC $(\mathrm{meq} / 100 \mathrm{~g})$ & Fases cristalinas (por DRX) \\
\hline $\mathbf{A B}$ & $59 \pm 2$ & Ilita, caulinita, feldspato e quartzo \\
$\mathbf{A P}$ & $187 \pm 5$ & Esmectita, feldspato e quartzo \\
\hline
\end{tabular}

Fluorescência de raios $X$ das argilas $A B F, A B$ e $A P$

A fim de determinar a concentração de elementos presentes nas argilas, utilizou-se a espectroscopia por fluorescência de raios $\mathrm{X}$, que é um método de análise para determinação quantitativa e qualitativa dos elementos. ${ }^{26,27}$ A Tabela 8 mostra os dados relativos à proporção dos elementos na argila da Bahia fracionada $(\mathbf{A B F})$, argila da Bahia fracionada e na tratada com ácido para eliminação do ferro não estrutural (AB) e na argila da Paraíba (AP). Os experimentos de fluorescência de raios $\mathrm{X}$ mostraram alguns elementostraços (concentração menor que $1 \%$ ) presentes nas argilas estudadas: $\mathbf{A B F}, \mathbf{A B}$ e $\mathbf{A P}$.

ABF: $\mathrm{Mn}, \mathrm{Co}, \mathrm{Ni}, \mathrm{Cu}, \mathrm{Zn}, \mathrm{Rb}, \mathrm{Sr}, \mathrm{Y}, \mathrm{Sb}, \mathrm{Sm}, \mathrm{Tb}, \mathrm{Hr}$

AB: $\mathrm{Na}, \mathrm{P}, \mathrm{Co}, \mathrm{Ni}, \mathrm{Cu}, \mathrm{Rb}, \mathrm{Sr}, \mathrm{Y}$

AP: $\mathrm{Na}, \mathrm{P}, \mathrm{S}, \mathrm{Cr}, \mathrm{Mn}, \mathrm{Ni}, \mathrm{Rb}, \mathrm{Sr}, \mathrm{Zr}, \mathrm{Pr}, \mathrm{Nd}$

Tabela 8. Teores dos elementos presentes na argila da Bahia fracionada (ABF) e na tratada com ácido (AB) e na argila da Paraíba (AP)

\begin{tabular}{lccc}
\hline Analito & $\begin{array}{c}\text { Concentração } \\
\text { na AP }(\%)\end{array}$ & $\begin{array}{c}\text { Concentração } \\
\text { na ABF }(\%)\end{array}$ & $\begin{array}{c}\text { Concentração } \\
\text { na AB }(\%)\end{array}$ \\
\hline- & 697,417 & 694,232 & 699.3 \\
- & 6,606 & 6,456 & 6,404 \\
$\mathrm{Na}$ & - & 0,844 & - \\
$\mathrm{Mg}$ & 2,395 & 1,253 & 1,114 \\
$\mathrm{Al}$ & 14,05 & 19,061 & 19,743 \\
$\mathrm{Si}$ & 66,116 & 62,073 & 63,503 \\
$\mathrm{P}$ & - & 0,161 & - \\
$\mathrm{K}$ & 0,377 & 2,621 & 2,629 \\
$\mathrm{Ca}$ & 0,934 & 0,71 & 0,318 \\
$\mathrm{Ti}$ & 0,7 & 0,783 & 0,849 \\
$\mathrm{Fe}$ & 8,723 & 5,876 & 5,285 \\
$\mathrm{Zr}$ & - & 0,061 & 0,057 \\
\hline
\end{tabular}

Destaca-se que os teores de ferro nas argilas $\mathbf{A B F}$ e $\mathbf{A B}$ são bastante semelhantes, indicando a presença de pequena quantidade de ferro como cátion interlamelar na argila fracionada ABF. Se o ferro existisse na argila $\mathbf{A B F}$ como cátion trocável, seria retirado no tratamento ácido e a quantidade de ferro na argila $\mathbf{A B}$ seria menor. Assim, conclui-se que o ferro está presente na estrutura cristalina dessas argilas. 


\section{Avaliação química por reação de cetalização da isatina}

A avaliação química do efeito da ativação por microondas na atividade catalítica das argilas foi realizada utilizando-se a reação de cetalização da isatina com etilenoglicol ${ }^{28}$ (Figura 7). Para isso empregou-se $1 \mathrm{mmol}$ de isatina $(147 \mathrm{mg}), 10 \mathrm{mmol}$ de etilenoglicol $(0,6 \mathrm{~mL})$ e $147 \mathrm{mg}$ de catalisador. Foram testados nove catalisadores: as argilas K10, AB e AP sem ativação (sem expoente, Tabela 9), as argilas ativadas termicamente (expoente T, Tabela 9) ou as argilas ativadas por microondas (expoente MO, Tabela 9). A mistura reacional foi irradiada com microondas por 15 min em potência de $72,7 \mathrm{~W}$.
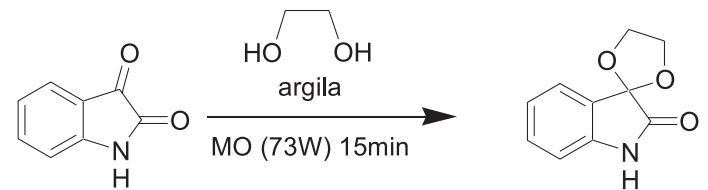

Figura 7. Reação de cetalização da isatina

Após os 15 min de irradiação com microondas, a mistura reacional foi filtrada, e o filtrado analisado por cromatografia gasosa (CG). A conversão da isatina, analisada por CG, é mostrada na Tabela 9. O rendimento do cetal preparado com esta metodologia e purificado variou entre 84 a $89 \%$.

Houve pequena redução na conversão da isatina quando utilizados os catalisadores ativados por microondas. O rendimento da cetalização aqui apresentado é superior ao do processo convencional com aquecimento térmico, ${ }^{29} \mathrm{e}$ a reação é bem mais rápida. Os valores de conversão da isatina em relação ao tipo de argila (AB, AP ou K10) são coerentes com a área específica das argilas.

Considerando que ilita é um argilomineral não expansível, de baixa área específica, de baixa capacidade de troca catiônica e sem cátions metálicos interlamelares, e considerando que a argila $\mathbf{A B}$ contém principalmente ilita, a baixa atividade catalítica desta argila pode ser justificada pela presença majoritária deste argilomineral. ${ }^{13,14}$ Como a reação estudada é catalisada por ácidos de Lewis ou de Bronsted, é possível inferir que, embora o catalisador K10 não apresente fases cristalinas bem definidas, apresenta um caráter ácido mais forte que o da argila da Bahia e da argila da Paraíba. Além disso, a área específica do catalisador K10 informada pelo fabricante $\left(240 \mathrm{~m}^{2} / \mathrm{g}\right)$ é cerca de três vezes maior que a das argilas naturais aqui empregadas.

Foi avaliada a conversão da isatina sem a presença do catalisador, nas mesmas condições descritas acima, ou seja, empregando-se 1 mmol de isatina $(147 \mathrm{mg})$ e $10 \mathrm{mmol}$ de etilenoglicol $(0,6 \mathrm{~mL})$ irradiados com microondas por 15 min em potência de 72,7 W. Nessas condições não se observou formação de cetais, por CG.

\section{PARTE EXPERIMENTAL}

\section{Determinação da potência do aparelho de microondas}

Foi utilizado aparelho de microondas doméstico marca Brastemp, modelo JetDefrost com capacidade de 27 L. Em um béquer de $2 \mathrm{~L}$ foi colocado $1 \mathrm{~L}$ de água (medido em um balão volumétrico de $1 \mathrm{~L}$ ). $\mathrm{O}$ aquecimento foi realizado por $120 \mathrm{~s}$, nos graus de potências disponíveis no aparelho (1 a 10). A potência (P) foi determinada pela razão entre a energia absorvida pela água $\left(\mathrm{nC}_{\mathrm{p}} \Delta \mathrm{T}\right)$ e o tempo de irradiação: $\mathrm{P}=\mathrm{nCp}(\Delta \mathrm{T}) / \mathrm{t}$, onde $\mathrm{n}$ é o núme- ro de moles de água (55,6 mols no caso); $\mathrm{C}_{\mathrm{p}}$ é a capacidade calorífica molar da água $\left(75,3 \mathrm{~J} \mathrm{~K}^{-1} \mathrm{~mol}^{-1}\right) ;^{9} \Delta \mathrm{T}$ é a variação de temperatura observada e t é o tempo de irradiação (120 s no caso estudado).

\section{Argilas utilizadas}

Montmorilonita K10, comercializada pela Sigma-Aldrich Corp., St. Louis, MO, USA. Trata-se de uma argila preparada inicialmente pela Süd Chemie a partir de uma montmorilonita natural, que é sujeita a calcinação a $873 \mathrm{~K}$, seguida de lavagem ácida. Esse tratamento destrói grande parte da estrutura cristalina característica da montmorilonita natural. Segundo dados do fornecedor (Aldrich), a K10 contém alumínio tetracoordenado e apresenta área específica, calculada por BET, de $240 \mathrm{~m}^{2} / \mathrm{g}$ e volume de microporos de $0,1 \mathrm{~mL} /$ g. Foram utilizadas também duas naturais: uma proveniente da Bahia - AB - e outra da Paraíba - AP. A argila da Bahia (AB) foi coletada às margens do Rio das Contas, no município de Jequié. Essa argila foi fracionada, fornecendo a argila $\mathbf{A B F}$. A argila $\mathbf{A B F}$ sofreu tratamento ácido para ativação e retirada de ferro livre, ${ }^{30}$ fornecendo a argila AB. A argila da Paraíba (AP) proveio da fazenda Bravo, município de Boa Vista, e foi cedida pelo Prof. V. Ferreira (UFF-RJ). A argila foi recebida já na granulometria na faixa de argilominerais. Devido à pequena quantidade de material disponível, essa argila não sofreu fracionamento ou tratamento ácido.

\section{Fracionamento da argila $\mathrm{ABF}$}

A $20 \mathrm{~g}$ da amostra seca, adicionou-se $100 \mathrm{~mL}$ de água destilada e $5 \mathrm{~mL}$ de hidróxido de sódio 1,0 mol/L (defloculante). A suspensão foi agitada por 2 min e deixada em repouso por $2 \mathrm{~h}$. Após o repouso, a amostra foi agitada novamente em agitador magnético por $15 \mathrm{~min}$. A suspensão foi passada através de uma peneira de 270 mesh (diâmetro de malha $=0,053 \mathrm{~mm}$ ). A fração retida na peneira foi denominada fração areia. O filtrado foi coletado em proveta de $1000 \mathrm{~mL}$ e a proveta foi avolumada em $1000 \mathrm{~mL}$. A mistura foi agitada vigorosamente por $2 \mathrm{~min}$ e deixada em repouso por $24 \mathrm{~h}$. Coletou-se os $20 \mathrm{~cm}$ superiores da suspensão contida na proveta. Esta fração foi denominada fração argila. A operação de coleta da suspensão rica em argila foi repetida 3 vezes. Após as coletas, adicionou-se 5,0 mL de $\mathrm{HCl}$ 1,0 $\mathrm{mol} / \mathrm{L}$, para neutralizar a solução e permitir a floculação da argila. $\mathrm{O}$ sólido foi centrifugado e lavado com água destilada até teste negativo com solução de $\mathrm{AgNO}_{3}$ 1,0 mol/L. Esse sólido foi denominado ABF. A fração silte, constituída basicamente de feldspato e mica, corresponde ao material depositado na proveta. ${ }^{31}$

\section{Tratamento ácido para ativação da argila $\mathrm{AB}$}

Tratou-se, sob agitação mecânica, $5 \mathrm{~g}$ da argila da Bahia fracionada (ABF) com ácido clorídrico a $10 \%(\mathrm{p} / \mathrm{v})$ a $90{ }^{\circ} \mathrm{C}$, sob refluxo, durante $2 \mathrm{~h}$. Lavou-se com água destilada o sólido obtido após esse tratamento. O sólido foi então colocado para secar a $55^{\circ} \mathrm{C}$ por $24 \mathrm{~h}$. O material sólido seco foi desagregado e peneirado até se obter frações menores que 200 mesh. ${ }^{12}$ Essa fração foi denominada $\mathbf{A B}$.

\section{Análise textural das argilas}

As análises texturais das amostras foram realizadas em equipamento Micromeritics A.S.A.P. 2010, após tratamento a vácuo, à temperatura

Tabela 9. Conversão da isatina com diferentes catalisadores

\begin{tabular}{lccccccccc}
\hline Argila & $\mathbf{K 1 0}$ & $\mathbf{K 1 0}^{\mathrm{T}}$ & $\mathbf{K 1 0}^{\mathrm{MO}}$ & $\mathbf{A B}$ & $\mathbf{A B}^{\mathrm{T}}$ & $\mathbf{A B}^{\mathrm{MO}}$ & $\mathbf{A P}$ & $\mathbf{A P}^{\mathrm{T}}$ & $\mathbf{A P}^{\mathrm{MO}}$ \\
\hline Conversão (\%) & $95 \pm 3$ & $99 \pm 2$ & $96 \pm 1$ & $76 \pm 5$ & $94 \pm 4$ & $89 \pm 4$ & $88 \pm 6$ & $98 \pm 3$ & $94 \pm 4$ \\
\hline
\end{tabular}


de $50{ }^{\circ} \mathrm{C}$ por $12 \mathrm{~h}$ para as argilas $\mathbf{A B}$ e $\mathbf{A B M O}$ e $18 \mathrm{~h}$ para as argilas $\mathbf{A P}$ e APMO, no próprio instrumento, e medidas por adsorção-dessorção de nitrogênio à temperatura do nitrogênio líquido. Para a determinação das áreas específicas, foi empregada a Equação BET.

\section{Difratometria de raios $\mathrm{X}$ das argilas}

Os difratogramas de raios $\mathrm{X}$ das amostras de argilas naturais e da K10, obtidos pelo método do pó, foram coletados em um difratrômetro Bruker-AXS D5005 equipado com espelho de Goeble para feixe paralelo de raios $\mathrm{X}$, nas seguintes condições de operação: radiação Co $\mathrm{K} \alpha(35 \mathrm{kV} / 40 \mathrm{~mA})$; velocidade de goniometro de $0,02^{\circ} 2 \theta$ por passo com contagem de $1,0 \mathrm{~s}$ por passo e coletados de 5 a $80^{\circ} 2 \theta$. A interpretação qualitativa foi efetuada por comparação com padrões contidos no banco de dados PDF02 (ICDD, 1996) em software Bruker Diffrac ${ }^{\text {Plus }}$.

\section{Ressonância magnética nuclear no estado sólido}

Os espectros de RMN-MAS de ${ }^{27} \mathrm{Al}$ foram obtidos em espectrômetro Bruker Avance, modelo DRX-300, operando em um campo magnético de 7,05 Tesla, com freqüência de Larmor de $78,2 \mathrm{MHz}$. As amostras foram acondicionadas em rotores de zircônia $\left(\mathrm{ZrO}_{2}\right)$ de $5 \mathrm{~mm}$ de diamêtro e giraram a $6 \mathrm{kHz}$. Foi usada seqüência de pulsos simples, com comprimentos de pulsos de 7 $\mu$ s e intervalo entre os pulsos (D1) de $0,3 \mathrm{~s}$. Os espectros foram obtidos após a realização de 5000 varreduras. Os deslocamentos químicos do ${ }^{27} \mathrm{Al}$ tiveram como referência uma amostra de $\mathrm{AlCl}_{3} \cdot 6 \mathrm{H}_{2} 0$ sólido $(0 \mathrm{ppm})$. Os espectros de RMN-MAS de ${ }^{27} \mathrm{Al}$ foram obtidos no mesmo aparelho, na freqüência do ${ }^{29} \mathrm{Si}$ de 59,6 $\mathrm{MHz}$, à temperatura ambiente e com excitação de pulsos simples. As amostras giraram em rotor de zircônia de $5 \mathrm{~mm}$ de diâmetro, à velocidade de rotação de $4 \mathrm{kHz}$. Os deslocamentos químicos de ${ }^{29} \mathrm{Si}$ foram referenciados utilizando um padrão secundário de caulin $(\delta=-91,5 \mathrm{ppm})$. Foi utilizado desacoplamento de alta potência de ${ }^{1} \mathrm{H}$ durante a aquisição do sinal de ${ }^{29} \mathrm{Si}$. Utilizou-se tempo de aquisição de $0,049 \mathrm{~s}$ e $\mathrm{PW}\left(90^{\circ}\right)$ de $8,1 \mathrm{~ms}$.

\section{Determinação da capacidade de troca catiônica (CTC) das argilas}

Cerca de $1 \mathrm{~g}$ do material (argila natural) foi pesado e trocado (através de 5 centrifugações de 5 min cada, a $3000 \mathrm{rpm}$ ) com 40,0 $\mathrm{mL}$ de solução de acetato de potássio $1,0 \mathrm{~mol} / \mathrm{L}$. A seguir, a suspensão foi lavada com etanol 95\% (5 centrifugações), desprezando-se sempre a solução sobrenadante. Após esta lavagem para a retirada do contra-íon, a amostra foi trocada com $40 \mathrm{~mL}$ de solução $1,0 \mathrm{~mol} / \mathrm{L}$ de acetato de amônio (5 centrifugações), coletandose as soluções sobrenadantes. A solução resultante foi avolumada em balão volumétrico de $100,0 \mathrm{~mL}$; dessa solução foi retirada uma alíquota de $0,5 \mathrm{~mL}$ e avolumada em balão volumétrico de $50,0 \mathrm{~mL}$. $\mathrm{O}$ teor de íons $\mathrm{K}+$ nesta solução foi determinado por espectrofotometria de chama, utilizando equipamento Micronal B262. O procedimento foi repetido três vezes. Utilizou-se a média das concentrações de íons $\mathrm{K}+$ obtidas por espectrofotometria de chama.

\section{Fluorescência de raios $X$ das argilas}

Foi utilizado um espectrômetro de fluorescência de raios $\mathrm{X}$ marca Philips, modelo PW2400. Para análise utilizou-se pastilha com amostra calcinada e fundida com tetraborato de lítio com massa inicial de amostra igual a 1,00 g. A massa inicial de amostra e fundente foi de $8,00 \mathrm{~g}$, obtendo-se 7,94 g para a massa final após calcinamento. Foram analisados os elementos do boro ao urânio. Para quantificação dos elementos presentes nas argilas utilizou-se o programa SQUANT 14", que acompanha o equipamento. A intensidade máxima do detetor com fluxo foi de $2000 \mathrm{kcps}$, e a do detetor por cintilação foi de $1000 \mathrm{kcps}$.

\section{Reação de cetalização da isatina}

A $1 \mathrm{mmol}$ da isatina $(147,13 \mathrm{mg})$ foi adicionada a mesma quantidade em massa da argila (K10, AB ou AP, não ativadas, ativadas termicamente ou ativadas por microondas). Foram então adicionados 5 mmols (aproximadamente $0,3 \mathrm{~mL}$ ) de etilenoglicol e a mistura reacional foi irradiada com microondas em potência mínima $(72,7$ $\mathrm{W}$ ) por $1 \mathrm{~min}$. Em seguida, foram adicionados mais $5 \mathrm{mmols}$ (aproximadamente $0,3 \mathrm{~mL}$ ) de etilenoglicol e a mistura reacional foi irradiada também em potência mínima por mais 14 min. A mistura reacional foi filtrada, lavada com água destilada e o produto da reação foi extraído com acetato de etila. A fase orgânica foi seca com sulfato de sódio anidro, filtrada e o solvente foi evaporado. O produto de reação foi analisado por CGAR.

\section{Dados do cetal da isatina}

$\mathrm{C}_{10} \mathrm{H}_{9} \mathrm{NO}_{3}$. Spiro[1,3-dioxolano-2,3'-indol]-2'(1'H)-ona. EM [70 $\mathrm{eV}, \mathrm{m} / \mathrm{z}$ (\%)]: 191 (12), 163 (100), 146 (4), 136 (4), 119 (57), 104 (6), 92 (25), 76 (8), 64 (7). IV ( $\left.\mathrm{KBr}, \mathrm{cm}^{-1}\right): 3428,3213,3115,2894$, 2846, 1741, 1622, 1474, 1217, 1085, 759, 622, 486. RMN ${ }^{1} \mathrm{H}(200$ $\mathrm{MHz}, \mathrm{CDCl}_{3}, \delta$ ppm): 4,28 a 4,40 (2H, m); 4,48 a 4,60 (2H, m); $6,82(1 \mathrm{H}, \mathrm{d}, \mathrm{J}=7,8 \mathrm{~Hz}) ; 7,04(1 \mathrm{H}, \mathrm{t}, \mathrm{J}=7,8 \mathrm{~Hz}) ; 7,27(2 \mathrm{H}, \mathrm{m}) ; 8,67$ (1H, sl). RMN ${ }^{13} \mathrm{C}\left(75 \mathrm{MHz}, \mathrm{CDCl}_{3}, \delta \mathrm{ppm}\right): 66,0 ; 102,6 ; 110,9$; 123,$4 ; 124,6 ; 125,2 ; 131,8 ; 142,0 ; 175,9$.

\section{CONCLUSÕES}

Por difratometria de raios $\mathrm{X}$ não são observadas alterações significativas na estrutura das fases cristalinas das argilas ativadas por microondas, entretanto houve pequena redução na área específica das argilas ativadas por microondas, estando, porém, as variações de área específica dentro do erro previsto para o método BET. Houve também pequena redução na conversão da isatina ( 2 a $5 \%$ ) quando utilizados os catalisadores ativados na irradiação de microondas, indicando que há uma pequena perda de atividade catalítica na ativação por microondas. É preciso considerar se é vantajosa a redução do tempo de ativação, de $1 \mathrm{~h}$ em aquecimento convencional para $10 \mathrm{~min}$ em microondas, tendo em vista a perda de atividade catalítica.

\section{MATERIAL SUPLEMENTAR}

São apresentados, em http: //quimicanova.sbq.org.br, os espectros de ressonância magnética nuclear das argilas, no estado sólido, para os núcleos de alumínio-27 e silício-29, na forma de arquivo PDF, com acesso gratuito.

\section{REFERÊNCIAS}

1. Rodrigues, M. G. F.; Pereira, K. R. O.; Valenzuela-Diaz, F. R.; Cerâmica 2006, $52,260$.

2. Lidstrom, P.; Tierney, J.; Wathey, B.; Westman, J.; Tetrahedron 2001, 57, 9225 .

3. Rosini, F.; Nascentes, C. C.; Nóbrega, J. A.; Quim. Nova 2004, 27, 1012.

4. Sanseverino, A. M.; Quim. Nova 2002, 25, 660.

5. Silva, F. C.; Ferreira, V. F.; Souza, M. C. B. V.; Quim. Nova 2006, 29, 376.

6. Varma, R. S.; Tetrahedron 2002, 58, 1235. 
7. Sadicoff, B. L.; Amorim, M. C. V.; Mattos, M. C. S.; Quim. Nova 2000, 23, 557.

8. Varma, R. S.; Green Chem. 1999, 1, 43.

9. Graebin, C. S.; Eifler-Lima, V. L.; Quim. Nova 2005, 28, 73.

10. Coelho, A. C. V.; Santos, P. S.; Santos, H. S.; Quim. Nova, 2007, 30, 1282.

11. Silva, C. R.; Nóbrega, J. A.; Blanco, T.; Quim. Nova 2005, 28, 137.

12. Laszlo, P.; Science 1987, 235, 1473.

13. Sieskind, O.; Albrecht, P.; Tetrahedron Lett. 1993, 34, 1197.

14. Kingston, H. M. Em Introduction to Microwave Sample PreparationTheory and Practice; Jassie, L. B., ed.; American Chemical Society: Washington, DC, 1988

15. Barboza, A. C. R. N.; Cruz, C. V. M. S.; Graziani, M. B.; Lorenzetti, M. C.; Sabadini, E.; Quim. Nova 2001, 24, 901.

16. Brunauer, S.; Deming, L. S.; Deming, E.; Teller, E.; J. Am. Chem. Soc. 1940, 62, 1723.

17. Doan, P. T.; Dissertação de Mestrado, Mississippi State University, USA, 2004.

18. Brunauer, S.; Emmett, P. H.; Teller, E.; J. Am. Chem. Soc. 1938, 60, 309.

19. Santos, P. S.; Ciência e Tecnologia de Argilas, Ed. Edgard Blücher Ltda.: São Paulo, 1989, vol. 1.

20. Shichi, T.; Takagi, K.; J. Photochem. Photobiol., C 2000, 1, 113.
21. Deleuze, M.; Goiffon, A.; Ibanez, A.; Philippot, E.; J. Solid State Chem. $1995,118,254$.

22. Miranda, R.; Ríos, H.; Delgado, F.; Castro, M.; Cogordán, A.; Salmón, M.; Appl. Catal., A 2003, 244, 217.

23. Fetter, G.; Tichit, D.; Menorval, L. C.; Filgueiras, F.; Appl. Catal., A 1995, $126,165$.

24. Jackson, M. L.; Soil Chemical Analysis, Prentice Hall of India Ltd.: India, 1967.

25. Frenkel, H.; Shainberg, I.; Soil Sci. Soc. Am. J. 1980, 44, 626.

26. Bertin, E. P.; Principles and Practice of X-Ray Spectrometric Analysis, Plenum Press: New York, 1970.

27. Muller, R. O.; Spectrochemical Analysis by X-Ray Fluorescence, Plenum Press: New York, 1972.

28. Silva, J. F. M.; Garden, S. J.; Pinto, A. C.; J. Braz. Chem. Soc. 2001, 12, 273.

29. Silva, R. B.; Dissertação de Mestrado, Universidade Federal do Rio de Janeiro, Brasil, 1990.

30. Foletto, E. L.; Volzone, C.; Morgado, A. F.; Porto, L. M.; Cerâmica 2002 47, 208.

31. Jackson, M. L.; Soil Chemical Analysis - Advanced Course, $2^{\text {nd }}$ ed., Wiscosin University, Dept. of Soil Science, E. U. A., 1974. 


\section{Angelo da Cunha Pinto}

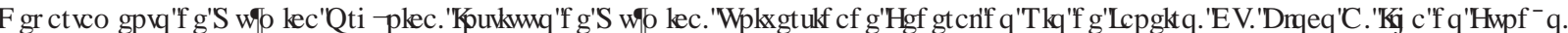
5 IRGH-DQHIR 5 - \%DNO

Cristiano Honório de Oliveira

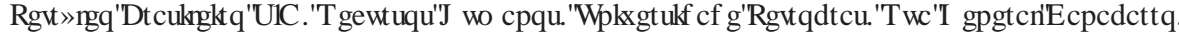

5 IRGH-DQHIR 5 - \%DVO

Núbia Moura Ribeiro*
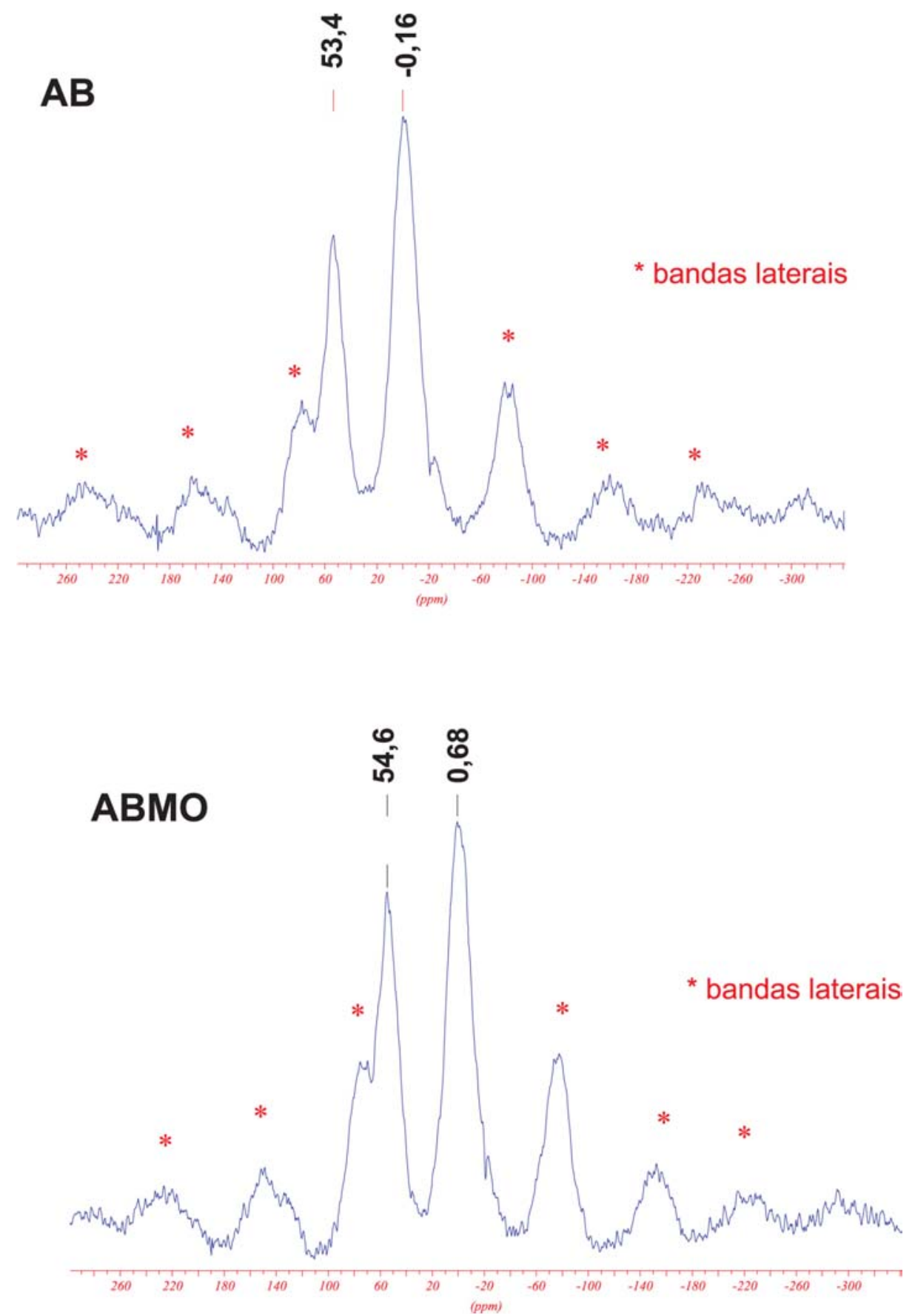

Figura 1S. Espectros de RMN-MAS de ${ }^{27}$ Al das argilas AB e ABMO 

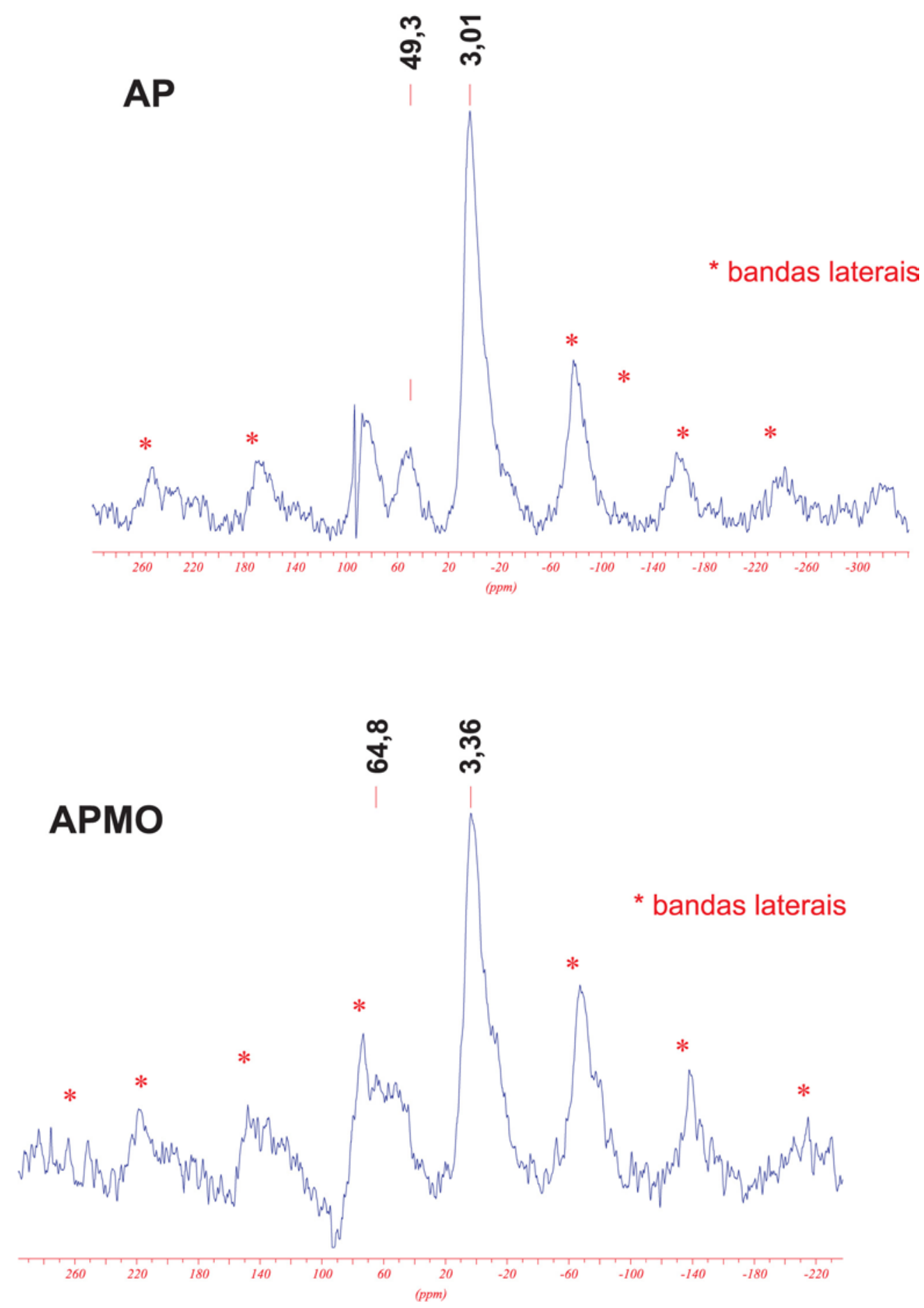

Figura 2S. Espectros de RMN-MAS de ${ }^{27} \mathrm{Al}$ das argilas AP e APMO 


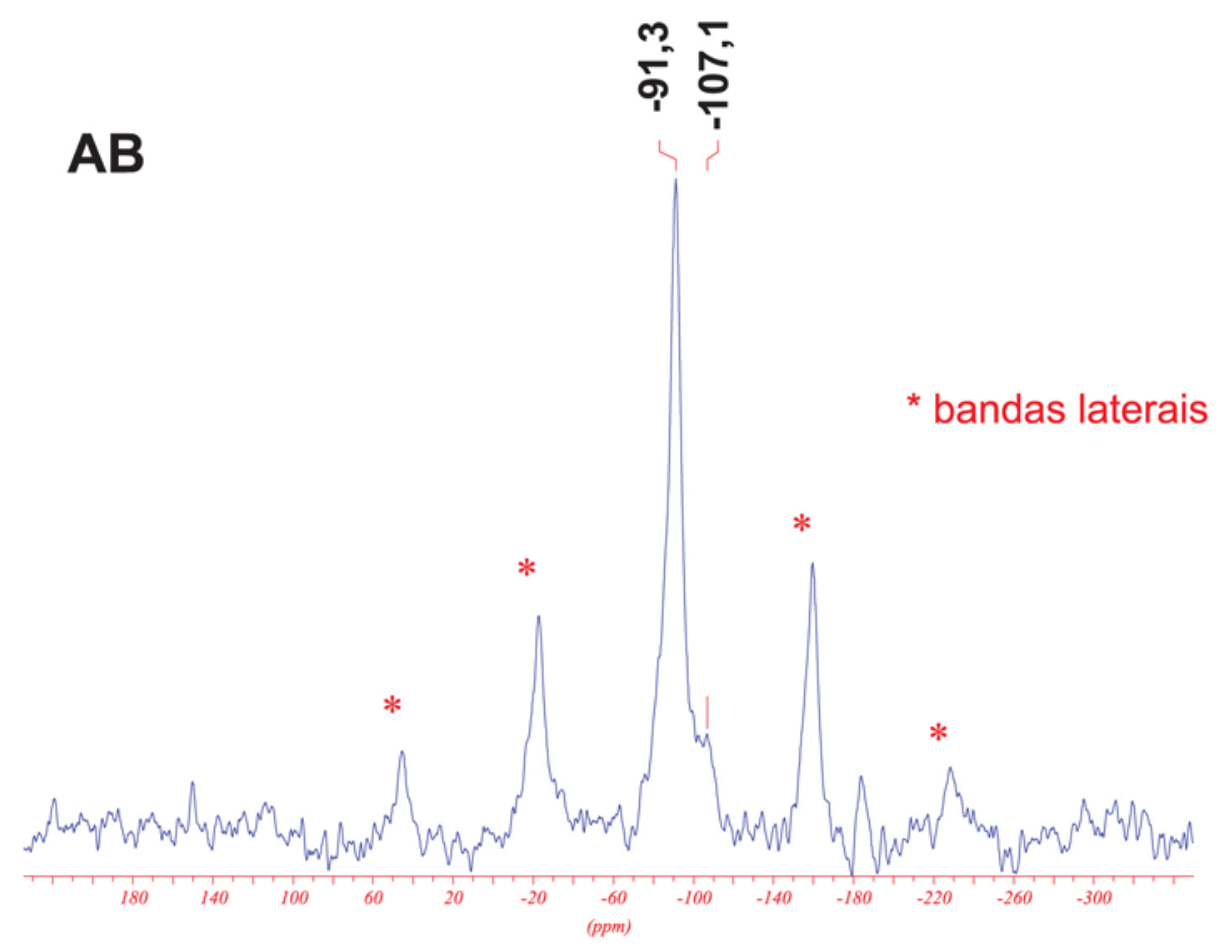

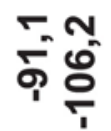

ABMO

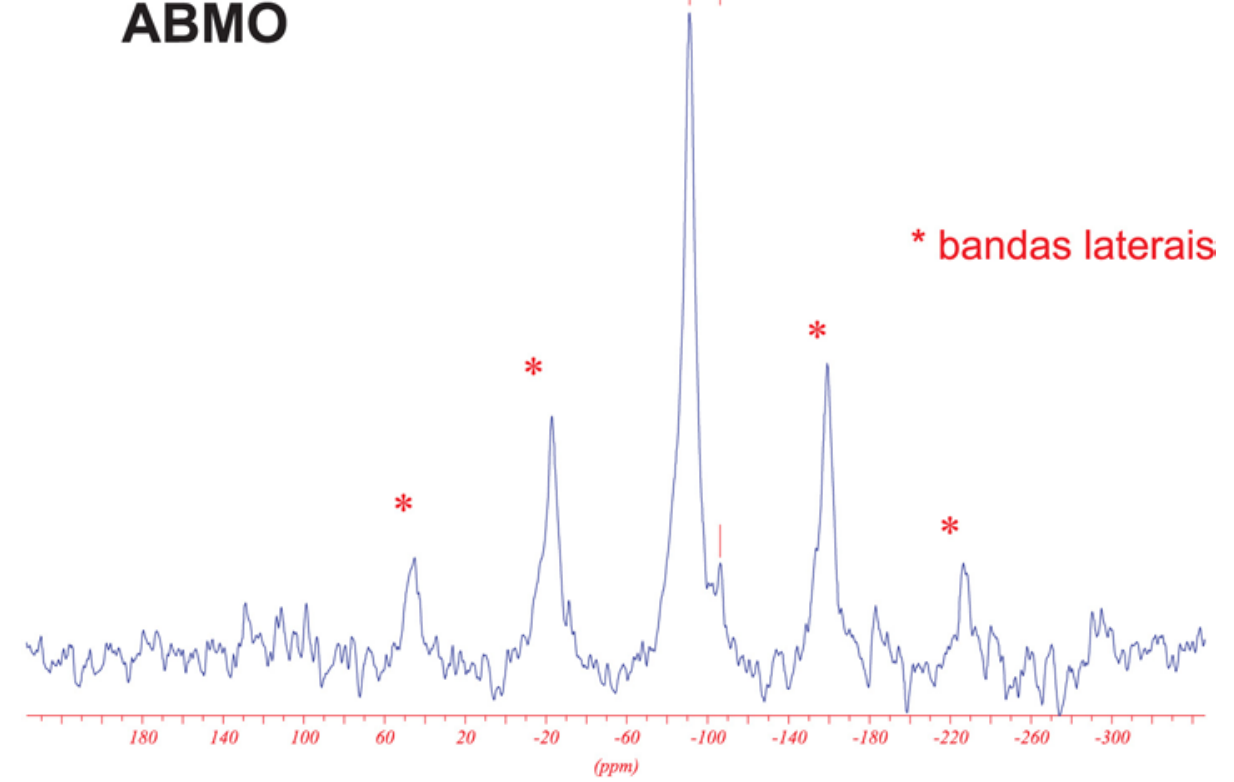

Figura 3S. Espectros de RMN-MAS de ${ }^{29} \mathrm{Si}$ das argilas AB e ABMO 


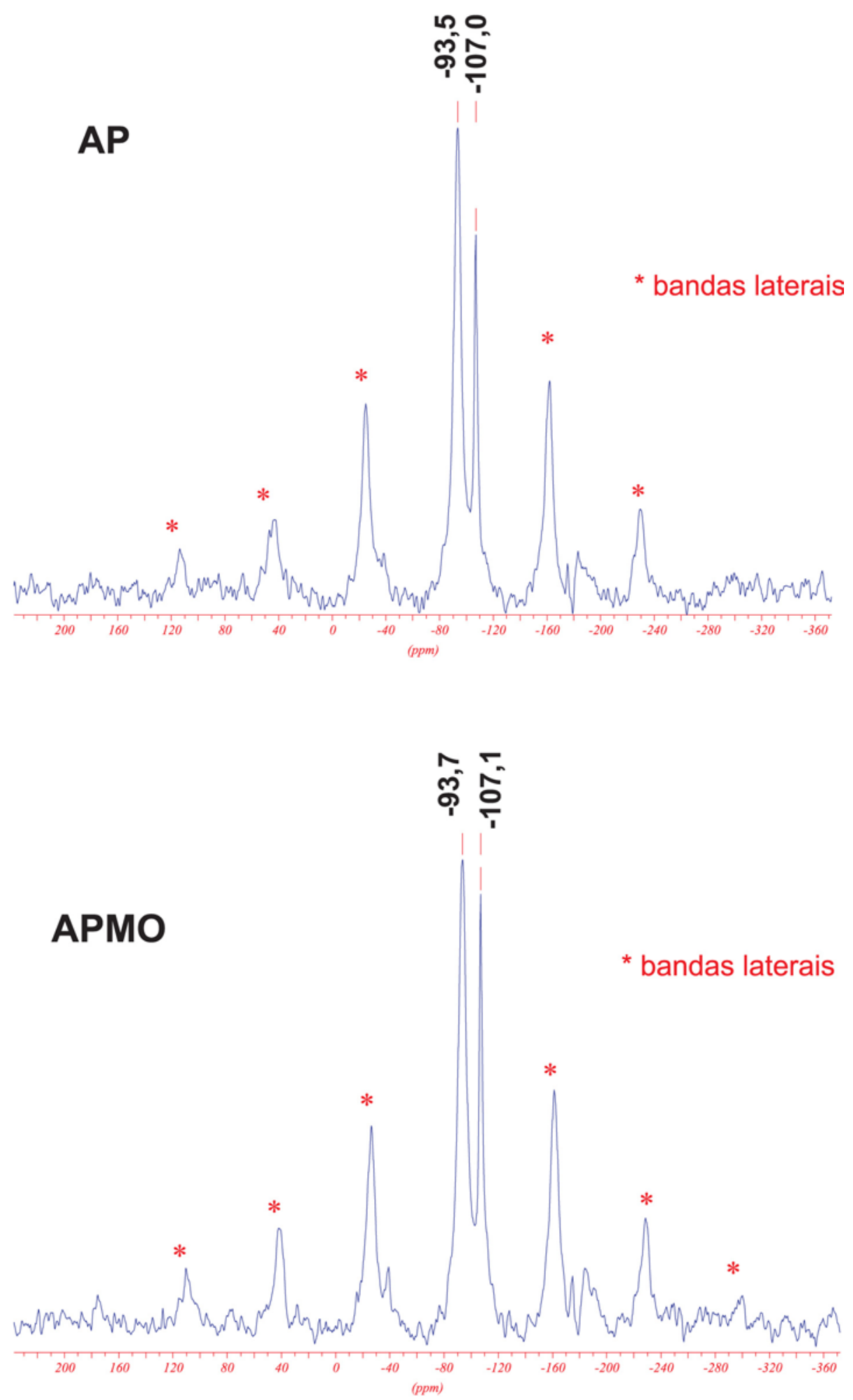

Figura 4S. Espectros de RMN-MAS de ${ }^{29}$ Si das argilas AP e APMO 